\title{
Prediction of wrinkling and springback in sheet metal forming
}

\author{
D.M. Neto ${ }^{1, a}$, M.C. Oliveira ${ }^{1}$, J.L. Alves ${ }^{2}$, A.D. Santos ${ }^{3}$ and L.F. Menezes ${ }^{1}$ \\ ${ }^{1}$ CEMUC, Department of Mechanical Engineering, University of Coimbra, Rua Luís Reis Santos, 3030-788 Coimbra, Portugal \\ ${ }^{2}$ CMEMS, Center for MicroElectroMechanics Systems, University of Minho, Campus de Azurém, 4800-058 Guimarães, Portugal \\ ${ }^{3}$ FEUP, Faculty of Engineering, University of Porto, Rua Dr. Roberto Frias, 4200-465 Porto, Portugal
}

\begin{abstract}
The finite element simulation is currently a powerful tool to optimize forming processes in order to produce defect-free products. Wrinkling and springback are main geometrical defects arising in sheet metal forming. Nevertheless, the prediction of such defects requires accurate numerical models. This study presents the experimental and numerical analysis of a rail with high tendency to develop both wrinkling (top surface of geometry) and springback (flange). The punch force evolution and the final geometry of the rail, evaluated in four different cross-sections, are the main variables analysed. Globally, the numerical results are in good agreement with the experimental measurements. However, the shape of the wrinkle is significantly influenced by the symmetry conditions considered in the model ( $1 / 4$ of the blank). In fact, considering the full model of the blank, the numerical results are in better agreement with the experimental ones. On the other hand, the computational cost of the numerical simulation considering the full blank is approximately 12 times higher than using $1 / 4$ of the blank.
\end{abstract}

\section{Introduction}

Sheet metal forming processes are commonly used in the automotive industry to produce several metal parts with complex geometry [1]. Currently, the major concerns of the automotive industry are the environmental protection (low fuel consumption and, consequently, low exhaust emissions) and the safety specifications. Accordingly, in order to meet these requirements, new materials have been introduced in car body manufacturing, such as highstrength steels as well as aluminium alloys [2-4]. Nevertheless, these material are more prone to develop geometrical defects, namely springback [5]. Another important geometrical defect arising in sheet metal forming is the wrinkling behaviour, which results from the instability under compressive stresses [6].

The integration of numerical simulation in the design and development of sheet metal forming processes was a key factor for the fulfilment of the increasing requirements for time and cost efficiency [7, 8]. In fact, the typical experimental trial-and-error die design has been progressively replaced by the finite element simulation of the process. This demands accurate numerical models, namely the introduction of new constitutive models [9], new types of finite elements [10] and the accurate treatment of the frictional contact conditions [11]. Despite the many advances in the numerical simulation tools development, the accurate prediction of geometrical defects still represents a challenge for the simulation [12]. For instance, it is known that wrinkling prediction is strongly affected by the finite element discretization [6]. Also, springback prediction is influenced by the model

\footnotetext{
a Corresponding author: diogo.neto@dem.uc.pt
}

selected to describe the material kinematic hardening behaviour [13].

This study presents the experimental and numerical analysis of a rail with a high tendency to develop both wrinkling (top surface of geometry) and springback (at the flange), thus representing an additional challenge to simulation, due to a possible interaction of effects. The experimental procedure is described in Section 2, while the adopted finite element code is presented in Section 3. The proposed numerical models of the forming process are defined in Section 4 and the comparison between numerical and experimental results is presented in Section 5. The main conclusions are outlined in Section 6.

\section{Experimental procedure}

This study deals with the sheet metal forming of a rail, which is prone to $2 \mathrm{D}$ springback behaviour and wrinkling on the top surface. In order to obtain a representative set of reference experimental results, required for validation the different strategies that may be adopted in the numerical simulation, special care was devoted to the standardization of the experimental procedure [14]. The experimental setup involves a punch, a die and a blank-holder, as shown in Figure 1. The forming process comprises three phases. In the first one, the die moves downward, clamping the blank between the die and the blank-holder with an initial $90 \mathrm{kN}$ prescribed force. This blank-holder force is defined by six nitrogen gas springs (see Figure 1), being all connected, in order to assure the same pressure in each of them. In the second phase, the die and blank-holder move 
downward together, forming the part and defining a punch penetration of $60 \mathrm{~mm}$. At this stage the gas springs are being compressed, thus increasing the blank-holder force from 90 to $130 \mathrm{kN}$. The third phase is the part removal from tools, in which the springback or elastic recovery takes place (see Figure 2).

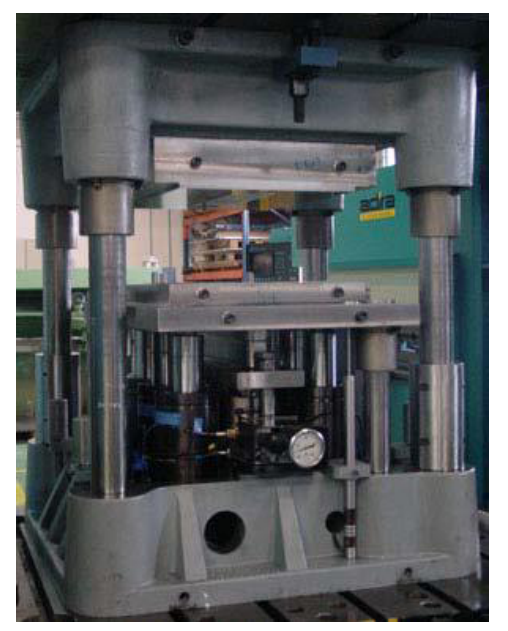

Figure 1. Experimental tool used to perform the forming process [14].

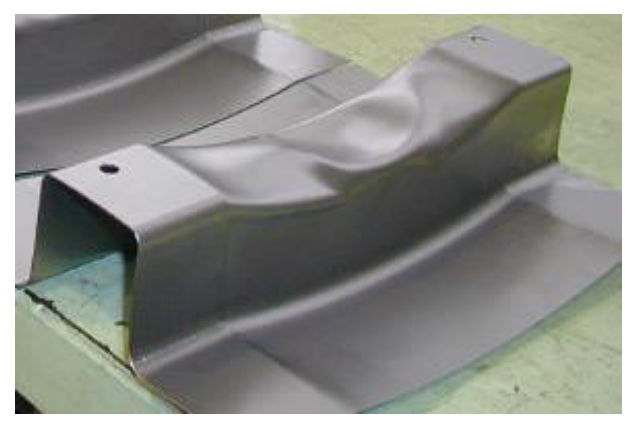

Figure 2. Experimental geometry of the rail after being removed from the forming tools. Two holes are trimmed on the top of the rail for fixing the part during its measurement.

The blank sheet (mild steel DC06) is a square with $300.0 \mathrm{~mm}$ length and $1.0 \mathrm{~mm}$ thickness. The experimental tests are carried out with the rolling direction of the blank sheet aligned with the longitudinal direction of the rail. In order to try to guarantee a correct amount of lubricant and its uniform distribution in the blank, the lubrication method suggested by Santos et al. [15] was adopted. The amount of lubricant (Quaker 6130) is defined as 1.4 $\mathrm{g} / \mathrm{m}^{2} /$ face.

The evolution of both the punch and the blank-holder force was recorded by load cells placed under each gas spring and under the punch. The punch displacement is experimentally evaluated through a displacement transducer. At least five tests were performed in order to check the reproducibility of the experiments. Accordingly, a representative result is selected, for each measurement under analysis. At the end of the second phase of the forming process, two holes are punched on the top of the rail, as seen in Figure 2. These holes are used to obtain a reference position in the jig, which was defined for experimental measurements. The definition of the coordinate system used in the evaluation of the crosssection profiles of the rail is coincident with one of the holes, as shown in Figure 3. For this geometry, four crosssection profiles are defined, which are marked in Figure 3. Five sets of measurements are performed for each crosssection at every $3 \mathrm{~mm}$ [14].

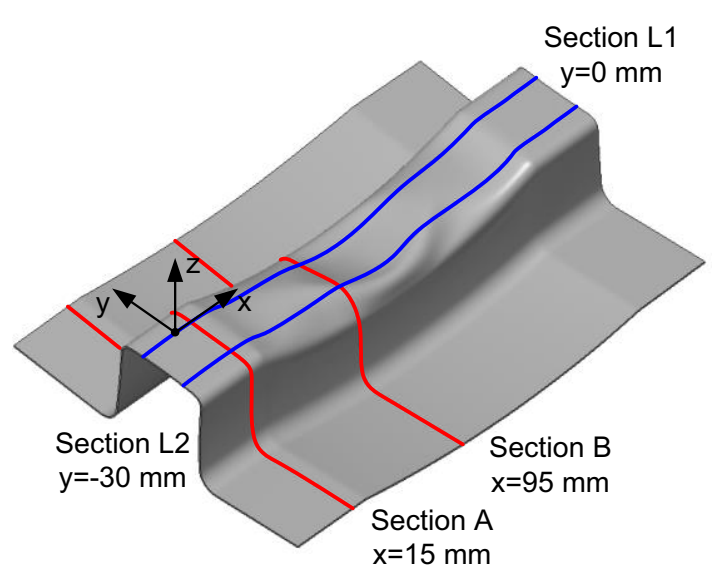

Figure 3. Definition of the four cross-sections used to evaluate experimentally and numerically the final geometry of the rail.

\section{DD3IMP - static implicit FE code}

The in-house static implicit finite element code DD3IMP [16], which has been specifically developed to simulate sheet metal forming processes, is adopted in the present study to carry out the numerical simulations. The mechanical model takes into account large elastoplastic strains and rotations, while the evolution of the deformation process is described by an updated Lagrangian formulation.

\subsection{Variational principle}

The rate form of the equilibrium equations and boundary conditions can be expressed by the principle of virtual velocities [17] in the form:

$$
\int_{V}\left\{\left(\dot{\boldsymbol{\sigma}}^{\mathrm{J}}-2 \boldsymbol{\sigma} \mathbf{D}\right): \delta \mathbf{D}+(\mathbf{L} \boldsymbol{\sigma}): \delta \mathbf{L}\right\} d V=\int_{S} \overline{\mathbf{t}} \cdot \delta \mathbf{v} d S,
$$

where $V$ denotes the domain occupied by the body and $S$ represents the surface on which the external forces $\overline{\mathbf{t}}$ are prescribed. $\delta \mathbf{v}$ denotes the virtual velocity field, $\mathbf{L}$ is the velocity gradient tensor and $\mathbf{D}$ is the strain rate tensor, which is the symmetric part of $\mathbf{L}$. The Jaumann derivative of the Cauchy stress tensor $\boldsymbol{\sigma}$ is given by:

$$
\dot{\boldsymbol{\sigma}}^{\mathrm{J}}=\dot{\boldsymbol{\sigma}}+\boldsymbol{\sigma} \mathbf{W}-\mathbf{W} \boldsymbol{\sigma},
$$

where $\dot{\sigma}$ stands for the time derivative of the Cauchy stress tensor and $\mathbf{W}$ is the total spin tensor (antisymmetric part of L).

\subsection{Constitutive material models}

The elastoplastic constitutive model adopted considers isotropic elastic behaviour and anisotropic plastic 
behaviour. The differential form of the constitutive equation is given by:

$$
\dot{\boldsymbol{\sigma}}^{\mathrm{J}}=\mathbf{C}^{\mathrm{ep}}: \mathbf{D}
$$

where $\mathbf{C}^{\text {ep }}$ is the fourth-order tensor that defines the elastoplastic modulus in the current configuration, according to the adopted constitutive model. The expression for this tensor depends of the algorithms adopted in the integration of the constitutive model and the type of relation considered between the states at the beginning and at the end of the loading increment. Thus, it is possible to consider the tangent elastoplastic modulus or the consistent elastoplastic modulus.

Several advanced yield criteria and work hardening laws (constitutive models) have been implemented in DD3IMP to allow a better description of the different material mechanical behaviour $[13,18]$. In each load step, an explicit approach is used to calculate the trial solution, which is adjusted using a generalization of the $r_{\min }$ strategy [19]. In order to guarantee the equilibrium of the deformable body, this trial configuration is successively corrected using an implicit method. The Newton-Raphson method is used to solve the nonlinear system of equations arising from the spatial and temporal discretization of the weak form. Some high performance computing techniques have been incorporated to take advantage of multi-core processors, namely OpenMP directives in the most time consuming branches of the code [20].

\subsection{Frictional contact conditions}

The modelling of the blank with solid elements allows the accurate evaluation of the contact forces and the throughthickness gradients (stress and strain) [21]. On the other hand, the forming tools are considered rigid and their outer surfaces are modelled by Nagata patches [22]. The friction between the blank and the forming tools is described by the classical Coulomb's law. The frictional contact problem is regularized through the augmented Lagrangian method [23], leading to a mixed system of equations involving both displacements and contact forces as unknowns [24]. The Newton-Raphson scheme is used to solve, in a single iterative loop, the non-linearities associated with both the contact and the elastoplastic behaviour of the deformable body [16].

\section{Numerical model}

In order to analyse the influence of the symmetry conditions on the wrinkling phenomena, two distinct finite element models are studied. The first one considers only $1 / 4$ of the blank due to geometric and material symmetry conditions of the forming process. On the other hand, the entire forming process is simulated in the second model. Further, in the full model, both the sheet and the rolling direction are rotated $1^{\circ}$ with the Ox direction, which induces non-symmetrical conditions on the forming process.

\subsection{Material behaviour}

The mechanical behaviour of the mild steel DC06 is assumed elastoplastic. The elastic behaviour is considered isotropic and constant, which is described by Hooke's law with Young's modulus of $210 \mathrm{GPa}$ and Poisson ratio of 0.30 . Regarding the plastic behaviour, the isotropic hardening is described by the Swift law, which is combined with kinematic hardening of ArmstrongFrederick type. The yield stress defined by the Swift law is given by:

$$
Y=K\left(\varepsilon_{0}+\bar{\varepsilon}^{\mathrm{p}}\right)^{n} \quad \text { with } \quad \varepsilon_{0}=\left(\frac{Y_{0}}{K}\right)^{1 / n},
$$

where $K, \varepsilon_{0}$ and $n$ are the material parameters, while $\bar{\varepsilon}^{\mathrm{p}}$ denotes the equivalent plastic strain. The kinematic part of the work hardening [25], i.e. the evolution of the backstress tensor $\mathbf{X}$, is described by:

$$
\dot{\mathbf{X}}=C_{\mathrm{X}}\left[\frac{X_{\text {sat }}}{\bar{\sigma}}\left(\boldsymbol{\sigma}^{\prime}-\mathbf{X}\right)-\mathbf{X}\right] \dot{\bar{\varepsilon}}^{\mathrm{p}} \quad \text { with } \quad \mathbf{X}(0)=\mathbf{0},
$$

where $X_{\text {sat }}$ characterizes the saturation value of $\mathbf{X}$, while the material parameter $C_{\mathrm{X}}$ characterizes the rate of approaching the saturation. The equivalent effective stress is denoted by $\bar{\sigma}$, while the deviator of the Cauchy stress tensor is represented by $\boldsymbol{\sigma}^{\prime}$.

The material parameters of the Swift law combined with kinematic hardening were identified using experimental results of a uniaxial tensile test as well as monotonic and Bauschinger simple shear tests, all of them performed with specimens cut along the rolling direction [26]. The five material parameters of the isotropickinematic hardening law are listed in Table 1. The adopted model provides a satisfactory fit of the experimental data (DC06), as highlighted in the stress-strain curves presented in Figure 4.

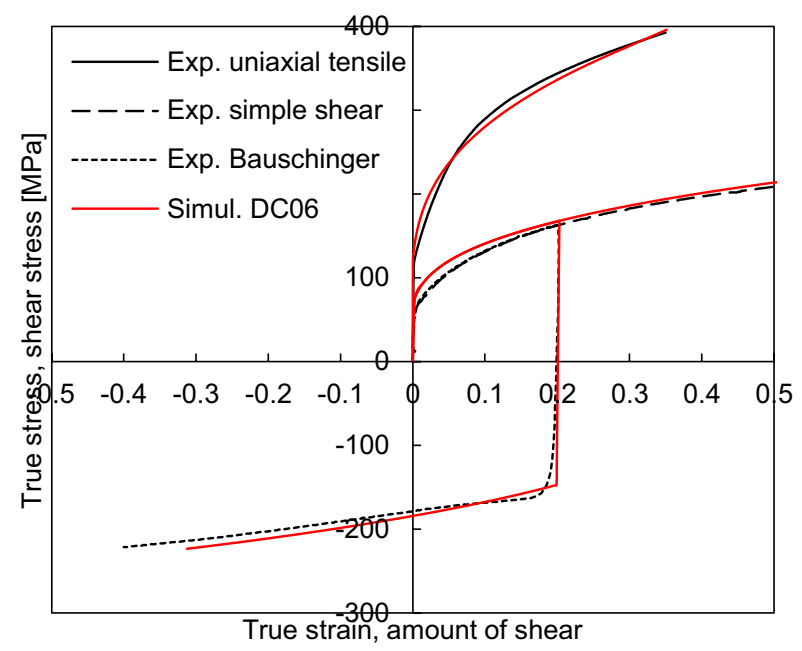

Figure 4. Comparison of the stress-strain curves predicted by the material model with the experimental ones for uniaxial tensile test, simple shear and Bauschinger simple shear after $20 \%$ forward shearing [26]. 
Table 1. Material parameters for the isotropic-kinematic hardening described by Swift law (DC06).

\begin{tabular}{|c|c|c|c|c|}
\hline$Y_{0}[\mathrm{MPa}]$ & $K[\mathrm{MPa}]$ & $n$ & $C_{\mathrm{X}}$ & $X_{\text {sat }}[\mathrm{MPa}]$ \\
\hline 122.2 & 435.0 & 0.219 & 1.45 & 116.7 \\
\hline
\end{tabular}

In order to deal with the anisotropic behaviour of the metal sheet, the Hill' 48 yield criterion and the associated flow rule are adopted. Accordingly, the equivalent effective stress is defined by the quadratic yield function:

$$
\begin{aligned}
\bar{\sigma}^{2}= & F\left(\hat{s}_{22}-\hat{s}_{33}\right)^{2}+G\left(\hat{s}_{33}-\hat{s}_{11}\right)^{2}+H\left(\hat{s}_{11}-\hat{s}_{22}\right)^{2}+ \\
& +2 L \hat{s}_{23}^{2}+2 M \hat{s}_{13}^{2}+2 N \hat{s}_{12}^{2},
\end{aligned}
$$

where $\hat{s}_{i j}$ stands for the components of the effective stress tensor in the axes of orthotropy. The six material constants $F, G, H, L, M$ and $N$ are calculated from the experimental $r$-values obtained by uniaxial tensile tests carried out at three different orientations with respect to the rolling direction. The obtained values are presented in Table 2 .

Table 2. Anisotropy parameters for the Hill' 48 yield criterion (DC06).

\begin{tabular}{|c|c|c|c|c|c|}
\hline$F$ & $G$ & $H$ & $L$ & $M$ & $N$ \\
\hline 0.264 & 0.283 & 0.717 & 1.500 & 1.500 & 1.279 \\
\hline
\end{tabular}

\subsection{Sheet and tools discretization}

The blank is discretized with 8-node hexahedral finite elements associated with a selective reduced integration scheme [27]. Since the prediction of wrinkling phenomena in sheet metal forming simulation requires a fine mesh [6], a regular mesh is adopted in the Oxy plane (element size of $1.0 \mathrm{~mm}$ in the central region), as shown in Figure 5. The discretization of the blank comprises two layers of finite elements through the thickness, which enable an accurate prediction of the forming forces and the springback [13]. Hence, the modelling of the full blank comprises 130,000 finite elements, while $1 / 4$ of the blank requires 32,500 finite elements (see Figure 5).

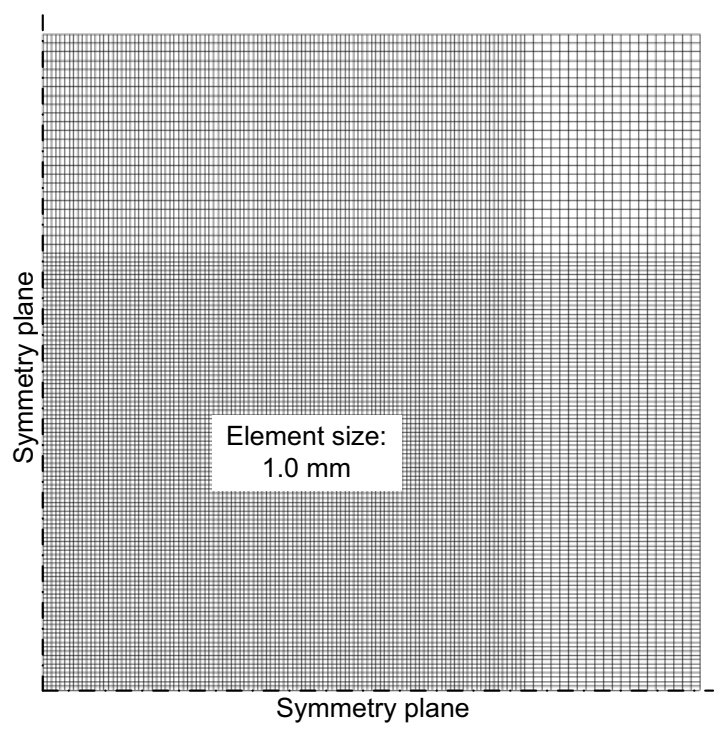

Figure 5. Discretization of the blank (1/4) using 32,500 hexahedral finite elements (2 layers through the thickness).
The geometry of the forming tools is modelled by Nagata patches [22], where the nodal normal vectors required for the contact surface smoothing approach are evaluated through the IGES file [28]. The discretization of the three tools involved in the numerical simulation is presented in Figure 6. The surface of the punch is described by 660 patches, the blank-holder comprises 300 patches and the surface of the die is discretized by 805 patches.

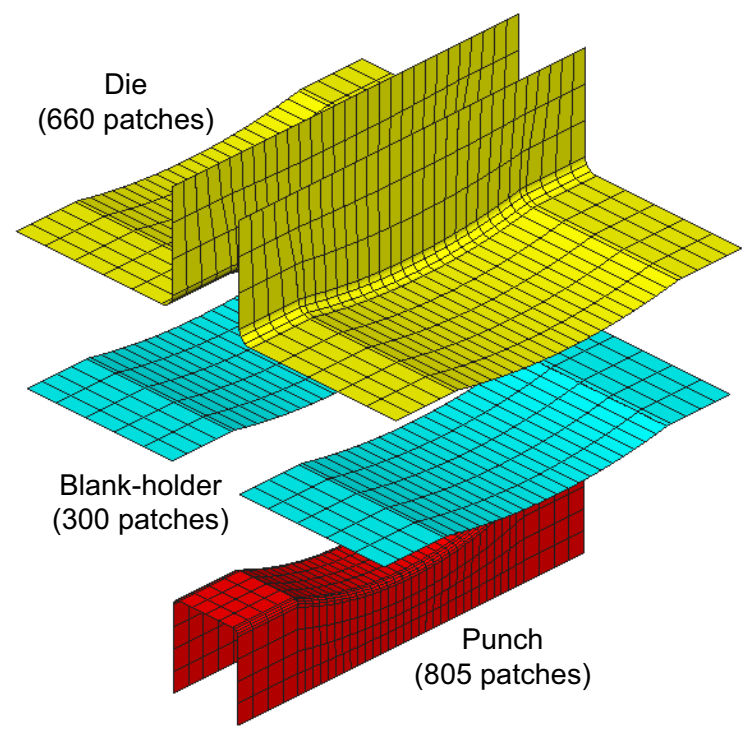

Figure 6. Discretization of the forming tools using Nagata patches.

\subsection{Friction model}

The friction coefficient is influenced by a wide set of parameters, such as: (i) micro and macro geometry of contacting surfaces; (ii) sliding velocity; (iii) contact pressure and (iv) temperature. Thus, an advanced friction models is adopted in the present study, where the friction coefficient $\mu$ is function of the normal contact pressure [29]. The evolutional law for Coulomb's friction coefficient is defined by a Hockett-Sherby law:

$$
\mu=B-(B-A) \exp \left(-m P^{n}\right)
$$

where $A, B, m$ and $n$ are numerical fitting parameters, while $P$ is the normal contact pressure.

The four parameters involved in the selected friction model (see Table 3) were determined using the flat die test [30]. The steel sample is maintained between two small flat dies, while the holding force is accurately controlled by load cells. The sliding speed is constant and equal to 1.5 $\mathrm{m} / \mathrm{min}$. The normal pressure is kept constant during the test. The flat dies were produced using the same steel selected for the forming tools and the lubrication conditions adopted are also the same (Quaker 6130 using $1.4 \mathrm{~g} / \mathrm{m}^{2} /$ face) [30]. Five pressure levels are considered during the tests, providing five different values of friction coefficient, as shown in Figure 7. The pressure strongly influences the friction coefficient. Indeed, the friction coefficient is greater at low pressure than at higher pressure. The fitting of the measured values by the 
advanced friction law is presented in Figure 7, showing a good agreement between them.

Table 3. Parameters of the adopted friction law for DC06.

\begin{tabular}{|c|c|c|c|}
\hline$A$ & $B$ & $m$ & $n$ \\
\hline 0.31 & 0.16 & 0.61 & 0.71 \\
\hline
\end{tabular}

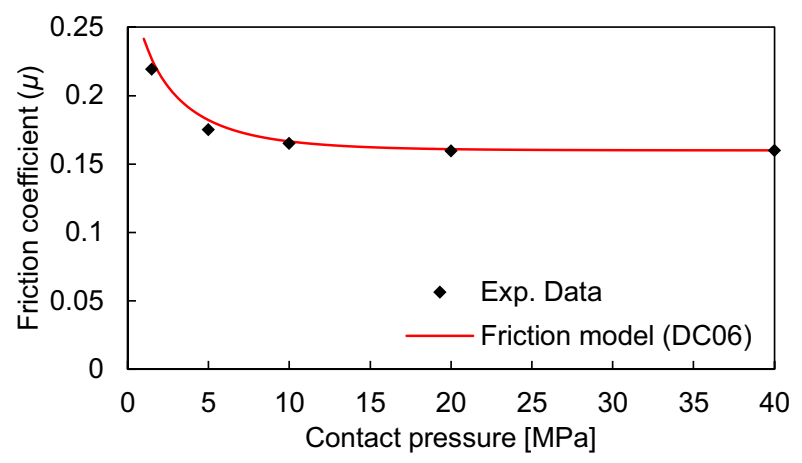

Figure 7. Evolution of the friction coefficient with the normal contact pressure, expressed by the adopted friction law. Comparison with experimental data from [30].

\section{Results and discussion}

The comparison between numerical and experimental results is presented in this section, namely the punch force evolution and the final geometry of the rail. The springback of the flange and the wrinkles on the top of the rail are the defects evaluated in the present study. Besides, the two finite element models are compared both in terms of accuracy and computational cost.

\subsection{Forming forces}

Both the punch and the blank-holder force evolutions are presented in Figure 8, comparing numerical and experimental results. The numerical blank-holder force increases linearly with the punch displacement, which is imposed in the numerical model, according with the experimental evolution. The predicted punch force evolution is identical for both finite element models presented. Nevertheless, the experimental value of the force is slightly overestimated by the numerical simulation, as shown in Figure 8. In fact, the initial slope predicted by the numerical simulation is higher than the one experimentally measured. The occurrence of wrinkling on the top surface of the rail induces a drop in the numerical force evolution at approximately $20 \mathrm{~mm}$ of punch displacement, which is more pronounced in the model considering the full blank.

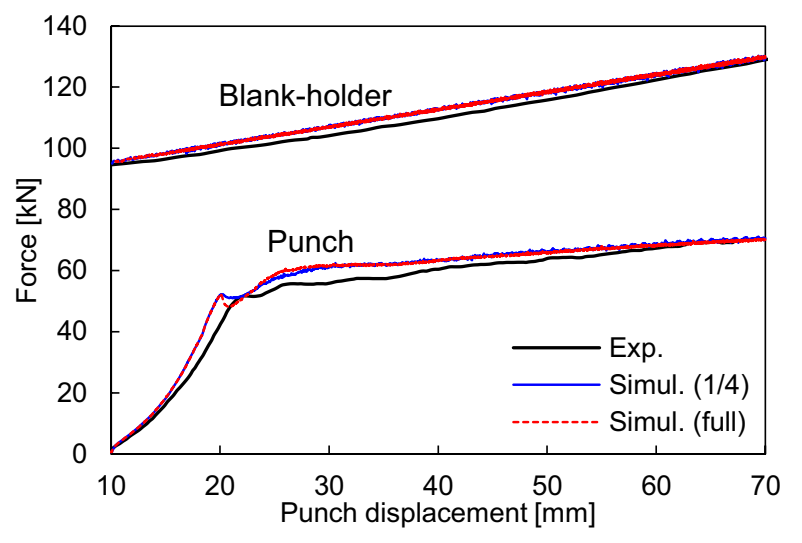

Figure 8. Comparison between experimental and numerical punch force evolution in the forming of the rail (DC06).

Considering the modelling of the entire blank, the total blank-holder force is equally divided by each flange of the rail. Thus, the vertical displacement of each flange of the blank-holder is adjusted independently in each increment, in order to guarantee a linear increase with the punch displacement. This procedure allow to obtain identical draw-in for each flange and, consequently, symmetry in the springback angle.

\subsection{Cross-section profiles}

The final geometry of the rail is evaluated through the four cross-section profiles defined in Figure 3, allowing to evaluate simultaneously the springback of the flange and the wrinkle on the top surface. The geometry of the rail after springback, predicted by the finite element simulation is shown in Figure 9. The numerical simulation of the entire blank provides an unsymmetrical wrinkle, which is accordance with the experimental observation (see Figure 2).

In order to assess the accuracy of each finite element model, the cross-section profiles obtained by numerical simulation are compared with the experimental ones. The comparison between experimental and numerical profiles in the cross-section $A(x=15 \mathrm{~mm})$ and $B(x=95 \mathrm{~mm})$ is presented in Figure 10 (a) and (b), respectively. Regarding the cross-section A, the numerical profile is in very good agreement with the experimental one. Besides, both numerical models (1/4 and full blank) provide identical results. Concerning the cross-section $\mathrm{B}$, the numerical profile is in good agreement with the experimental one, excluding the top surface of the rail, where the two numerical models predict distinct geometries (see Figure 10 (b)). In fact, the wrinkle predicted by numerical simulation is symmetric using $1 / 4$ of blank (see Figure 9 (a)), while it is unsymmetrical using the full model of the blank, which in accordance with the experimental result. Nevertheless, the geometry of the wrinkle is not perfectly defined, as shown in Figure 10 (b). Since the material adopted presents a clear anisotropic distribution of the $r$ values (see Table 2), a slight rotation the rolling direction with the symmetric full blank provides an identical wrinkle shape. 


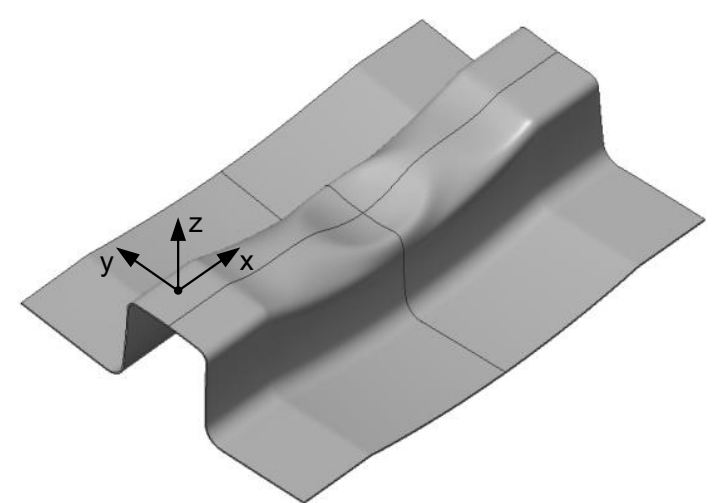

(a)

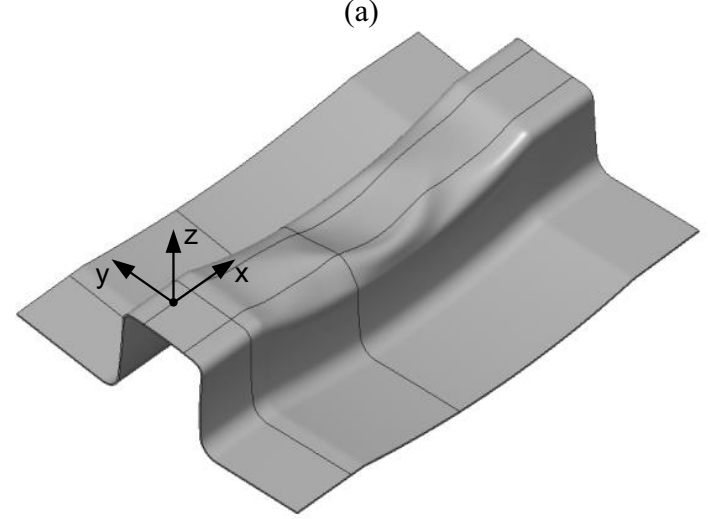

(b)

Figure 9. Final geometry of the rail after springback predicted by finite element simulation: (a) 1/4 of blank; (b) full blank including the four cross-sections used to evaluate the geometrical defects.

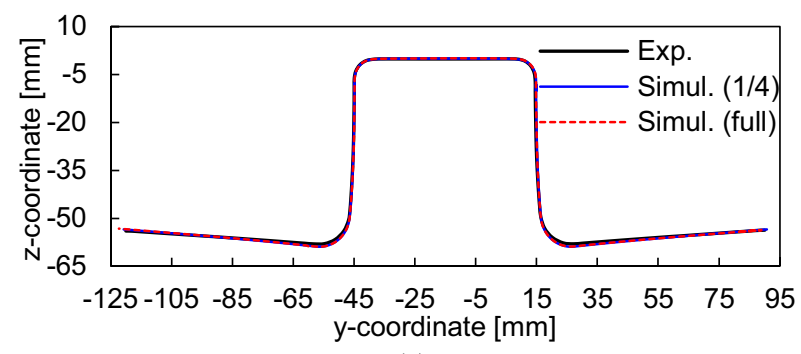

(a)

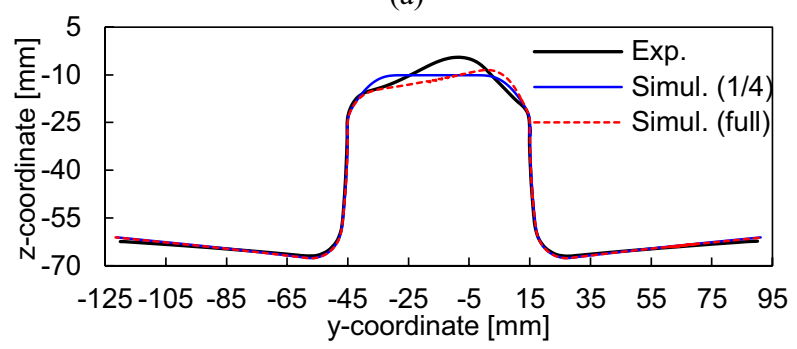

(b)

Figure 10. Comparison between experimental and numerical profile of the cross-section: (a) A ( $x=15 \mathrm{~mm})$; (b) $B(x=95 \mathrm{~mm})$.

The springback of the rail is quantified in this study by the angle between the flange and the horizontal plane. The comparison between experimental and numerical values is shown in Table 4, for both cross-sections previously studied. Since the rail after springback is rather symmetric (see Figure 10), the presented values for numerical model with the full blank are the average of the two flanges. The flange springback angle is slightly overestimated by the numerical simulation, as shown in Table 4. The difference is about $0.5^{\circ}$ in the cross-section $\mathrm{A}$ and $1.1^{\circ}$ in the crosssection B. Nevertheless, both numerical models provide identical results, as shown in Table 4 . Thus, the prediction of the flange springback behaviour is not considerably influenced by the shape of the wrinkle arising on the top surface.

Table 4. Comparison between experimental and numerical (full blank) springback angle of the flange.

\begin{tabular}{|c|c|c|c|}
\hline Cross-section & Exp. $\left[^{\mathbf{0}}\right.$ ] & 1/4 model $\left[{ }^{\mathbf{0}}\right.$ ] & Full model $\left[{ }^{\mathbf{0}}\right]$ \\
\hline A & 3.9 & 4.39 & 4.40 \\
\hline B & 4.3 & 5.41 & 5.37 \\
\hline
\end{tabular}

The profile of the rail measured in the cross-sections L1 and L2 (longitudinal direction) is directly dictated by the wrinkle arising on the top surface. The comparison between experimental and numerical profiles in the crosssection $\mathrm{L} 1(\mathrm{y}=0 \mathrm{~mm})$ and $\mathrm{L} 2(\mathrm{y}=-30 \mathrm{~mm})$ is presented in Figure 11 (a) and (b), respectively. Globally, the numerical predictions are in good agreement with the experimental measurements. On the other hand, the numerical shape of the rail is significantly influenced by the symmetry conditions adopted in the finite element model (1/4 model). Indeed, considering the full model of the blank, the numerical results are in better agreement with the experimental measurements, as shown in Figure 11.

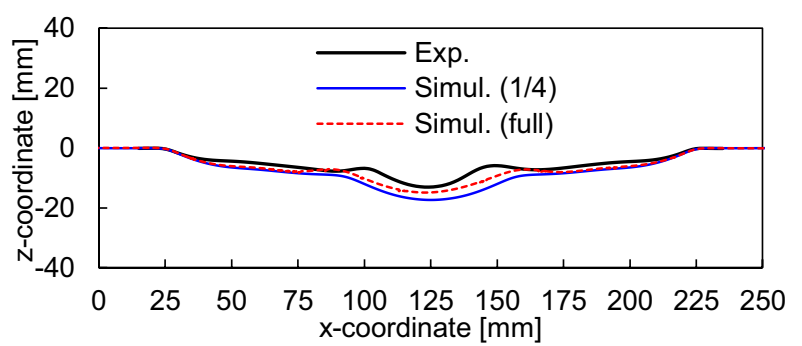

(a)

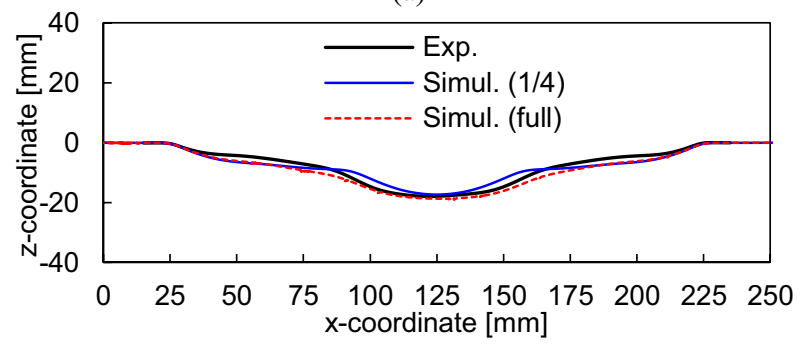

(b)

Figure 11. Comparison between experimental and numerical profile of the cross-section: (a) L1 (y=0 mm); (b) L2 (y=-30 mm).

\subsection{Computational performance}

The computational performance of the proposed finite element models is presented in Table 5. The adoption of the numerical model that takes into account the full blank leads to a significant increase of both the computational time and the number of increments. In fact the computational cost of the numerical simulation considering the full blank is approximately 12 times higher 
than using $1 / 4$ of the blank. The same behaviour is observed for the number of increments, as shown in Table 5 . This increase of the computational cost is a consequence of the higher number of finite elements adopted and the numerical complexity required to control the blank-holder force independently in each flange.

Table 5. Computational performance of both finite element models.

\begin{tabular}{|c|c|c|}
\hline & 1/4 model & Full model \\
\hline $\mathrm{N}^{\mathrm{o}}$ increments & 1823 & 4839 \\
\hline Computational time $[\mathrm{h}]$ & 30.4 & 384.7 \\
\hline
\end{tabular}

\section{Conclusions}

The application of the numerical simulation in sheet metal forming processes has been a key factor for the fulfilment of the increasing requirements for time and cost efficiency, as well as quality improvement. This study presents the experimental and numerical analysis of a rail prone to $2 \mathrm{D}$ springback and wrinkling defects. The finite element results are compared with the experimental ones in order to assess the accuracy on the proposed numerical models. The punch force evolution and the final geometry of the rail, evaluated in several cross-sections, are the main variables under analysis.

Two distinct finite element models are compared in this work, both in terms of accuracy and computational cost. Due to geometric and material symmetry conditions, only one quarter of the blank is considered in the first model. On the other hand, the second model takes into account the entire blank, which is rotated $1^{\circ}$ relative to the forming tools. Both numerical models provide identical punch force evolutions, as well as flange springback angles. However, these two variable are slightly overestimated by the numerical simulation. Regarding the wrinkle arising on the top surface of the rail, its shape is predicted differently by each finite element model. In fact, considering the full model of the blank, the numerical results are in better agreement with the experimental measurements, where the wrinkle tend to be unsymmetrical. Nevertheless, the computational cost of the numerical simulation considering the full blank is approximately 12 times higher than using 1/4 of the blank.

\section{Acknowledgements}

The authors gratefully acknowledge the financial support of the Portuguese Foundation for Science and Technology (FCT) under projects with reference UID/EMS/00285/2013, PTDC/EMS-TEC/0702/2014 (POCI-01-0145-FEDER-016779) and PTDC/EMSTEC/6400/2014 (POCI-01-0145-FEDER-016876) by UE/FEDER through the program COMPETE2020. The first author is also grateful to the FCT for the Postdoctoral grant SFRH/BPD/101334/2014.

\section{References}

1. A. Makinouchi, J. Mater. Process. Technol. 60, 19-26 (1996)

2. R. Kuziak, R. Kawalla, S. Waengler, Arch. Civ. Mech. Eng. 8, 103-117 (2008)

3. W.S. Miller, L. Zhuang, J. Bottema, A.J. Wittebrood, P. De Smet, A. Haszler, A. Vieregge, Mater. Sci. Eng. A 280, 37-49 (2000)

4. M.K. Kulekci, Int. J. Adv. Manuf. Technol. 39, 851865 (2007)

5. H. Laurent, J. Coër, P.Y. Manach, M.C. Oliveira, L.F. Menezes, Int. J. Mech. Sci. 93, 59-72 (2015)

6. D.M. Neto, M.C. Oliveira, R.E. Dick, P.D. Barros, J.L. Alves, L.F. Menezes, Int. J. Mater. Form. (to be published)

7. A.E. Tekkaya, J. Mater. Process. Technol. 103, 14-22 (2000)

8. G. Gantar, T. Pepelnjak, K. Kuzman, J. Mater. Process. Technol. 130-131, 54-59 (2002)

9. B. Revil-Baudard, O. Cazacu, P. Flater, N. Chandola, J.L. Alves, J. Mech. Phys. Solids 88, 100-122 (2016)

10. R. Alves de Sousa, J. Yoon, R. Cardoso, R. Valente, J. Gracio, Int. J. Plast. 23, 490-515 (2007)

11. D.M. Neto, M.C. Oliveira, L.F. Menezes, Arch. Comput. Methods Eng. (to be published)

12. M. Banu, M. Takamura, T. Hama, O. Naidim, C. Teodosiu, A. Makinouchi, J. Mater. Process. Technol. 173, 178-184 (2006)

13. M.C. Oliveira, J.L. Alves, B. Chaparro, L.F. Menezes, Int. J. Plast. 23, 516-543 (2007)

14. A.D. Santos, J. Ferreira Duarte, A. Reis, A. Barata da Rocha, L. Menezes, M. Oliveira, A. Col, T. Ono, J. Mater. Process. Technol. 125-126, 798-805 (2002)

15. A.D. Santos, A. Reis, J.F. Duarte, P. Teixeira, A.B. Rocha, M.C. Oliveira, J.L. Alves, L. Menezes, J. Mater. Process. Technol. 155-156, 1980-1985 (2004)

16. L.F. Menezes, C. Teodosiu, J. Mater. Process. Technol. 97, 100-106 (2000)

17. R.M. McMeeking, J.R. Rice, Int. J. Solids Struct. 11, 601-616 (1975)

18. D.M. Neto, M.C. Oliveira, J.L. Alves, L.F. Menezes, Mater. Des. 60, 368-379 (2014)

19. Y. Yamada, N. Yoshimura, T. Sakurai, Int. J. Mech. Sci. 10, 343-354 (1968)

20. L.F. Menezes, D.M. Neto, M.C. Oliveira, J.L. Alves, AIP Conf. Proc. 1353, 1220-1225 (2011)

21. C. Teodosiu, D. Daniel, H.-L. Cao, J.-L. Duval, J. Mater. Process. Technol. 50, 133-143 (1995)

22. D.M. Neto, M.C. Oliveira, L.F. Menezes, J.L. Alves, Comput. Methods Appl. Mech. Eng. 271, 296-320 (2014)

23. P. Alart, A. Curnier, Comput. Methods Appl. Mech. Eng. 92, 353-375 (1991)

24. A. Heege, P. Alart, Int. J. Numer. Methods Eng. 39, 165-184 (1996)

25. J. Lemaitre, J.-L. Chaboche, Mechanics of Solid Materials, Cambridge University Press (1990)

26. H. Haddadi, S. Bouvier, M. Banu, C. Maier, C. Teodosiu, Int. J. Plast. 22, 2226-2271 (2006)

27. T.J.R. Hughes, Int. J. Numer. Methods Eng. 15, 14131418 (1980) 
28. D.M. Neto, M.C. Oliveira, L.F. Menezes, J.L. Alves, Finite Elem. Anal. Des. 72, 35-46 (2013)

29. M.C. Oliveira, J.L. Alves, L.F. Menezes, Arch. Comput. Methods Eng. 15, 113-162 (2008)

30. P. Wouters, D. Daniel, C. Magny, Selection and Identification of Friction Models for the 3DS Materials-Identification of the Frictional Behaviour, Digital Die Design Systems (3DS) IMS 1999000051 , 2002. 